\title{
Universal Semantics in Translation
}

\author{
Zhenying Wang \\ School of Arts and Laws, China University of Mining and Technology \\ Ding 11 Xueyuan Road, Haidian District, Beijing 100083, China \\ Tel: 86-10-6283-6828Ｅ-mail: zhenyingwang2001@yahoo.com.cn
}

\begin{abstract}
What and how we translate are questions often argued about. No matter what kind of answers one may give, priority in translation should be granted to meaning, especially those meanings that exist in all concerned languages. In this paper the author defines them as universal sememes, and the study of them as universal semantics, of which applications are also briefly looked into.
\end{abstract}

Keywords: Universal semantics, Translation, Universal sememe

\section{Introduction}

Professionals in translation have been setting standards, demonstrating skills and designing courses for this work, with a purpose in mind to protect newcomers from "falling over the parapet into limbo"(Firth, 1957, p.197). However, do the protected get to know clearly how to translate or what to translate, after a long odyssey through experiences or academic training and having maintained considerably satisfactory performance? It might be hard to answer such questions even if they are asked of some of the protectors themselves in that the process of translation is far from an exclusively formal one of seeking after a series of equivalence at various levels and then lining up the materials in another order. The latter surely proves to be preliminary and helpful for cultivating translators, but cannot be so for cultivating translation theories. One of the further steps approaching translation as a branch of science is to identify the nature and laws in it, which surely will intensify translation training ultimately.

\section{The Role of Meaning in Translation}

When the ancient Romans of the third century BC translated from Greek and the former Chinese of Tang Dynasty translated from Sanskrit, they meant to obtain knowledge about cultures of other peoples speaking different languages, not to appreciate the exotic accent itself. And we know that the carrier of knowledge is meaning and that of meaning more often than not some more concrete things. When we start to explore a language, it is usually "from the standpoint of how it creates and expresses meaning." (Halliday, 2004, p.19) Though it seems that meaning is so vague, insubstantial, and elusive that it is impossible to come to any clear, concrete, or tangible conclusions about it, meaning usually is the only thing that may survive the handling of a tertiary translator. Therefore, it is more touchable than anything else for such a research to start with.

Then what do we mean by translation? By its nature, translation refers to a kind of transference between linguistic forms, or process of putting it "in other words" as Mona Baker (2000, p.53) pictured it. Generally, translation in a narrow sense is taken as interlingual transference from one language into another, while in a broad sense can be inter-symbol transference from one form whether linguistic or nonlinguistic into another. Theoretically and mathematically, translation activities may be regarded as computational process, and the transference may take the form of functional formulas as follows.

Formula 1: $\mathrm{M}=\mathrm{f}(\mathrm{L})$

Formula 2: $L^{\prime}=f^{\prime}(M)$

In the first formula, $\mathrm{M}$ stands for meaning, $\mathrm{L}$ for source language, and $\mathrm{f}$ is the set of formal rules for source language. In the second formula, $\mathrm{L}$ ' sands for target language, $\mathrm{M}$ for meaning, and $\mathrm{f}$ ' is the set of formal rules for target language. And $M$ remains the same in both formulas. The whole course of the two formulas in a sequence as $f(L) \Rightarrow M \Rightarrow f^{\prime}(M)$ $\Rightarrow$ L' represents the realization of M's formal transference from L into L'. The value domain of Formula 1 equals the definition domain of Formula 2; the definition domain of Formula 1 and the value domain of Formula 2 overlap with each other. The context of this sequence, i.e. the process of translation, is the union set of the definition domain of Formula 1 and the value domain of Formula 2. In condition that L equals L', $f$ and $f$ are opposite functions mutually. One point that deserves our attention is that the above-mentioned functions will not necessarily give a unique value for a single variable and by no means hold a feature of monotony for connections between formulations and meanings of language are always so complex and multi-hiberarchied, no mater within one or among more languages, that no one-to-one-valued mapping will exist.(Note: According to the assumptions, there is at least one value for each variable.) 
Henceforward in this research, ideal translation will be venturously defined as the alternation between linguistic formulations that carry the same meaning, the object of translation as various forms in natural languages, and the role of meaning in translation as that of settings and criterions. Take Picture 1 as an illustration of the ideal transference process. As we know, the course of translation may be logically divided into three phases, just for the sake of analytic convenience, i.e. analysis phase, transfer phase, and generation phase. In a perfect product of translation meaning is

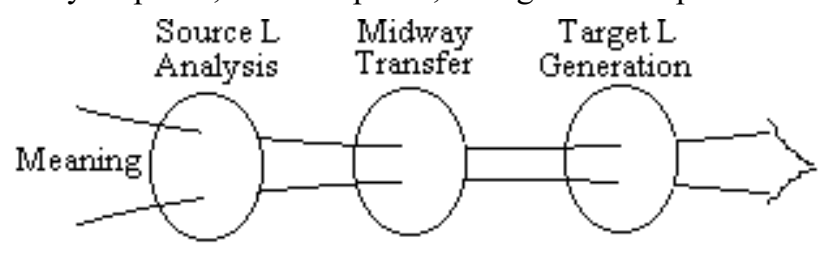

Picture 1 : Perfect Transltion

bound to have survived these three phases, and it is omnipresent in such a process.

\section{Universal Semantics}

In fact meaning does not indwell omnipresently in the process of translation for no other reason than being arbitrarily defined as, and on the contrary it is one part of the immediate aftermath of semantic universality. In accordance with social properties of language, the synchronic linguistic appearance comes directly from the combination of individual linguistic vectors, which have magnitude and direction. In theory there has to be a common exerting point for every two

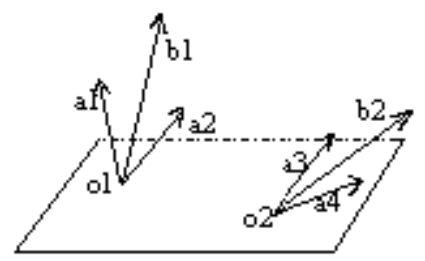

\section{Picture 2: Syuchrouic Plane of Lauguage}

vectors, and consequently all the vector exerting points form a synchronic plane that is relatively quiescent during the course of language development (See Picture 2). In Picture 2 a1, a2, a3 and a 4 stand for some of the individual vectors, b1 and b2 are some of the compositional vectors, and o1 and 02 represent some of the exerting points. It is absolutely on the basis of the universal connotational acceptability from this synchronic plane that people speaking the language are able to perform linguistic communications.

However, translation activities are intercultural as well as interlingual, so settings of them will not be as easy to identify. Yet it sounds reasonable that there is also a hypothetical synchronic plane for different languages in our mind when undertaking the task. Connotations of the semantic items belonging to this plane will be the same or similar, and readers of our translation products will apprehend the spirit of other cultures, provided that we form the accurate plane as comprehensive basis. (Coincidentally people speaking pidgin seem to have something like this in mind unconsciously.) Therefore the substantial basis of translation is universality in languages, not the universality of formulations or patterns, but that of most semantic items. Thus the author hereby takes the liberty of putting forward a bold idea concerning translation — Universal Semantics.

Everything under the sun in this world experiences events of birth, growth, decline and death consecutively, which is surely true of language. All languages, no matter natural or artificial, come into being and evolve little by little as a result of certain requirements from its users, and have disappeared or will withdraw from people's linguistic realm upon the extinction of requirements or the users themselves. But human language as a whole has always been evolving along with the development of human society, from the time when the first string of sounds that carried meaning was out of somebody's mouth. During the course of this evolvement the decisive and dominant role is at all time played by the pragmatic needs of human beings, which turned out to be semantic components when reflected in the concrete objectivity of language. The same or similar subjective and objective conditions, namely the psyche unity of mankind and the substantial unity of external environment, for the origination and development of various languages, lead to the common foundation of their existence, and this foundation in turn puts forth universal connotations in all languages.

As early as in the seventeenth century Descartes, Pascal, Leibniz and some other rationalistic thinkers had already begun the discussion on semantic primitives. In recent years a group of linguists including Zolkovskij and Wierzbicka systematically proposed a theory about natural semantic primitives for meta-language. The theory starts with the search for semantic primitives, which stand for those concepts "which can be understood without any definitions, and in terms of which all other concepts can be defined" (Wierzbicka, 1992, p.403). Examples for semantic primitives are words like "you", "I", and "this", which are difficult to be interpreted or defined. In fact, even if we managed to do this, the interpretation or definition would be either a vicious circle or a fuzzy explanation. And the objective of the research ab 
initio is to "avoid circularity" in linguistic interpretation (Geeraerts, 1994, p.3804). Although the set of semantic primitives in the so-called meta-language is so small a collection that its size has not been agreed on, the theory, together with its universal grammar, namely the valency of semantic primitives, contains considerable potentiality in linguistic description and interpretation. The proposal of this theory about semantic primitives stands out as a great breakthrough in the development of semantics and linguistics. And the primitives might well be regarded as the fascinating original variables in the linguistic axiom system that have been longed for by many western linguists, if further tested by practice. Nevertheless, it would be difficult to apply such theory naturally to the analysis of everyday lingual exchanges. No semantic research could afford leaving out the complexity of natural meaning, whereas the meta-language theory doubtlessly widens the gap between simple and compound semantic terms by interpreting all the semantic terms with semantic primitives, and consequently makes the work of language processing much more complicated for translation.

Actually, besides a core composed of primitives, among various natural languages there are still many other commonly shared items that are relatively more complicated than and can be defined or interpreted with semantic primitives. These items will be labeled as universal sememes in this paper, and the meaning contained in universal sememes labeled as universal meaning or universality, which lies universally in all kinds of languages and composes the formerly mentioned synchronic plane. All this may serve as the theoretical foundation of universal semantics.

Universal meaning refers to mental linguistic reflection of all matters, events, states, procedures and manners by language subjects, usually by human brains. When accommodated by varied componential units, e.g. phonemes, lexicons, phrases, clauses, sentences, and discourses, of different layers in language, universal meaning takes the forms of universal sememes of different levels. Higher-leveled universal sememes are composed of lower-leveled universal sememes, but the former are absolutely not equal to sheer mix-ups of the latter. Actually they are monolithic combinations of lower-leveled ones fabricated together as integrative ingredients. For instance, compounds, phrases and sentences are all constructed in this way. The semantic value of a universal sememe of higher level can be equal to, larger or smaller than, or definitely different from the sum-up semantic value of all its composing lower-leveled ones. And as a result, the combining patterns, by which higher-leveled sememes are produced, or grammar rules as traditionally called, are provided with a capacity of carrying meanings. Therefore we may divide fundamental universal sememes into two classes, i.e. conceptual and structural universal sememes. Conceptual universal sememes are lingual abstractions from single matters, events, states, procedures or manners, while structural universal sememes are abstractions from lingual relations between matters, events, states, procedures and manners. A conceptual universal sememe is a set of external lingual forms representing the same universal meaning, e.g. $\{\mathrm{I}$, Я, わたし\}, \{America, Америка, アメリ功; a structural universal sememe is just a standard name for a structural pattern, e.g. \{noun\}, \{subject\}, \{sequence\}, so on and so forth. Higher-leveled or compound universal sememes all consist of some fundamental ones from the two classes, of which conceptual ones are segmental and independent, while structural ones are attached to conceptual ones, hence dependent and super-segmental. However, some universal sememes may take different forms in different languages, for example, some structural sememes of tense and question in English may turn out to be conceptual ones of temporal and interrogative empty words when occurring in Chinese.

Furthermore, universality isn't a definite notion, but a rather fuzzy concept, since it would sound quite irrational if we regard the meaning that can be located in 100 kinds of natural languages as universal and meanwhile deny that in 99 this title. Alternatively we may conceive that the meaning in 100 is certainly more universal than one only in 99 . All universal sememes make up a fuzzy set, which takes all the semantic items that occur in human language as elements. When we regard human language as the universal background for discussion, namely the universal domain $\mathbf{U}$, and the collection of meaning-carrying linguistic units as a fuzzy subset $\mathbf{A}$ of $\mathbf{U}$, then elements of Set $\mathbf{A}$ can be determined by a mapping function $\boldsymbol{u}$ that stands for the diversity in degree of universality, and Mapping $\boldsymbol{u}$ is the membership function of Fuzzy Set A on Domain U. For this function, the definition domain is the collection of all semantic items in human language, and the value domain is $(0,1]$. Hereafter in this paper universal sememes may be defined in a mathematical way as follows:

Definition: Given a mapping $\boldsymbol{u}$ on the universal domain $\mathbf{U}$, and

$\boldsymbol{u}: \mathbf{U} \longrightarrow(0,1]$,

thus $\boldsymbol{u}$ fixes a fuzzy subset of $\mathbf{U}$, noted as $\mathbf{A}$. And $\boldsymbol{u}$ is called the membership function of $\mathbf{A}$, noted as $\boldsymbol{u} \mathrm{A}(\mathrm{x})$, which indicates the qualification of one element $\mathbf{x}$ as a member of Set $\mathbf{A}$.

Since it is likely that $\boldsymbol{u}$ assumes all values continuously in the half-open and half-closed zone of $(0,1]$, all semantic items that exist in human language are universal sememes of certain degree of universality. To what kind of degree an item is universal is determined by the corresponding value of the membership function with the item itself as the variable. The most universal semantic items are those with 1 as their membership value, mainly including: semantic primitives, e.g. $\{$ you $\},\{I\},\{$ this $\}$, and $\{$ that $\} ;$ most semantic terms representing human common cognition, e.g. \{high $\}$, $\{$ low $\},\{$ red $\},\{$ black $\},\{\operatorname{man}\}$, and $\{\operatorname{dog}\} ;$ proper nouns, e.g. $\{$ Beijing $\},\{\mathrm{UN}\},\{$ Thomas Edison $\}$, and $\{$ Fujisan\}; some 
structural universal sememes, e.g. \{subject\}, \{agent\}, \{passiveness\}, and \{adjective $\}$. The least universal are those semantic items that occur only in one kind of language, such as the words Eskimos use to describe the colors of snow melting to different extent, the membership value of which is close to zero. And membership values of other universal sememes are irregularly distributed between those of the two above-mentioned particular cases.

\section{Foundation of Translation}

It is one of the most important premises for all translation activities to acknowledge the universality of some meanings in natural languages. Although transference based on the fuzzy set $\mathbf{A}$ has proved one indispensable procedure for the whole process, we actually take a certain subset of $\mathbf{A}$ as groundsill instead of translating on the basis of set $\mathbf{A}$ as a whole. If translation takes place among all human languages, i.e. $\mathbf{U}$ serves as the universal domain, the most adaptive foundation should be one of A's subset, noted as A1, which consists of all elements whose membership value is 1 , for reason that all the other elements can be defined or interpreted as combinations of these fundamental ones in some given patterns. Now, if translation takes place among $\mathbf{n}$ languages $(n=2,3,4, \ldots)$, in turn the most adaptive foundation of translation will become more extensive. It then includes not only the elements whose membership value is 1 also some ones universal only in these languages and with a membership value less than 1 , and we may note this subset of $\mathbf{A}$ as $\mathbf{A} \lambda(\lambda<1)$, e.g. A0.9, A0.8, etc. When translating among certain number of languages, we need not know about the semantic universality of any other language or even its existence in this world, so the universal domain is no longer the set $\mathbf{U}$ of all human languages but a subset of $\mathbf{U}$, and in accordance the previous interceptive set $\mathbf{A} \lambda$ of $\mathbf{A}$ becomes $\mathbf{A} 1$ in the new universal domain. Elements of $\mathbf{A} 1$ occur in all concerned languages, they are the basic ingredients of them.

\section{Conclusions}

Universal semantics may find its practical significance in many fields, such as translation training, arts of translation, machine translation (Tennant, 1981, p.21), and even L2/3 acquisition. As for translation training and arts, we suggest a course emphasized both the target and source languages, and a method of interpreting in source language beforehand. In regard to machine translation and L2/3 acquisition, meaning-centered approaches might be preferable.

\section{References}

Baker, M. (2000). In Other Words: A Coursebook on Translation. Routledge and Foreign Language Teaching and Research Press.

Firth, J. R. (1957).. The Semantics of Linguistics Science. Papers in Linguistics 1934-1951. London: Oxford University Press.

Geeraerts, D. (1994). Semantic Primitives. In R.E. Asher (Eds.), The Encyclopedia of Language and Linguistics, Vol. 7:3804. Oxford: Pergamon Press.

Halliday, M.A.K. (2004). An Introduction to Functional Grammar. London: Arnold Publishers, Hodder Headline Group.

Tennant, H. (1981). Natural Language Processing. New York: Petrocelli Books Inc.

Wierzbicka, A. (1992). Semantic Primitives. In W. Bright (Eds.) International Encyclopedia of Linguistics, Vol. 3:403. New York: Oxford University Press. 